\title{
System Lifetime and Performance Improvement in Wireless Sensor Networks by having Improved Energy Distributed Unequal Clustering Protocol
}

Ashok T ( $\square$ ashoksubaash@gmail.com )

KNCET: Kongunadu College of Engineering and Technology

Prabakaran $\mathbf{R}$

University College of Engineering, BIT Campus, Anna University, Trichy

\section{Research Article}

Keywords: Wireless Sensor Network, Unequal Clustering, System lifetime, Energy consumption, Sensor Nodes, Packet loss ratio, Packet received ratio.

Posted Date: June 28th, 2021

DOl: https://doi.org/10.21203/rs.3.rs-582778/v1

License: (c) (i) This work is licensed under a Creative Commons Attribution 4.0 International License.

Read Full License 


\title{
System Lifetime and performance improvement in wireless sensor networks by having improved energy distributed unequal clustering protocol
}

\author{
Ashok T ${ }^{1}$ \\ ashoksubaash@gmail.com \\ Associate Professor in Department of Biomedical Engineering, Kongunadu College of \\ Engineering and Technology, Thottiam, Tiruchirappalli, Affiliated to Anna University - Chennai, \\ Tamilnadu \\ R.Prabakaran ${ }^{2}$ \\ hiprabakaran@gmail.com \\ Assistant Professor in Dept of Electrical and Electronics Engineering, University College of \\ Engineering, BIT Campus, Anna University, Tiruchirappalli, Tamil Nadu
}

\begin{abstract}
Wireless Sensor Network (WSN) is becoming a very important area of research in today's world and contributes a lot in the field of technology. Reducing energy consumption and improving the network lifetime is the key factor to be considered.Clustering provides an effective way for prolonging the lifetime of a wireless sensor network. Current clustering algorithms usually utilize two techniques, selecting cluster heads with more residual energy and rotating cluster heads periodically, to distribute the energy consumption among nodes in each cluster and extend the network lifetime. Also, it comprises various sensor nodes to detect different parameters. Among those non-replaceable batteries plays a greater part. Hence the system with such networks is essential that the sensor nodes consume as little energy as possible.To address the problem, we propose anovel model namely enhanced energy distributed unequal clustering which is mainly utilized for tackling energy consumption issues in multi-hop remote sensor systems. In the proposed method with an area of base station and energy are given significance as clustering parameters. Because of these parameters, diverse nodes are assigned. Here, another methodology has been proposed to enhance the working of EDUC, by electing cluster heads considering several nodes in the neighborhood. The incorporation of the area data for calculation of the opposition radii gives better adjusting of energy in correlation with the current methodology. The technique utilized is of holding similar bunches for a couple of rounds and is successful in decreasing the clustering overhead. The execution of the proposed convention has been assessed under three distinct scenarios and contrasted and existing conventions through reenactments. The outcomes demonstrate that the proposed plan beats the current conventions regarding system lifetime and performances in all the scenarios in terms of delay, energy consumption, packet loss ratio, and packet received ratio.
\end{abstract}

Keywords: Wireless Sensor Network, Unequal Clustering, System lifetime, Energy consumption, Sensor Nodes, Packet loss ratio, Packet received ratio. 


\section{Introduction}

Remote sensor systems are described by numerous asset limitations, for example, vitality, handling force, stockpiling, and transmission. Out of these components, the vitality of conveyed sensors has been the real asset imperative of the remote sensor systems. Part of research work has been done in the most recent decade to address this test [1, 2]. WSNs are sent thickly for information gathering applications including a lot of zones, for example, agribusiness, timberlands, coal mines, observing rail burrows, checking off a sun-based photovoltaic cell in a network, and so on. The base station (BS) is put far from the detecting field in the greater part of the cases. In such systems, information is assembled occasionally by the Base Station. Bunching with various leveled topologies is observed to be effective for acknowledging constant checking systems. It is shown that grouping the system offers more noteworthy life expectancy than the system with direct information transmission. It is demonstrated that the system life expectancy gets enhanced by a factor of around 2 or multiple times with bunching. There are numerous preferences of utilizing grouping conventions in information gathering systems. In a thick system, typically there is a vast volume of activity among the sensors, which prompts production of impedance and accordingly results in crashes. Normally, gathering the sensors would limit the number of long separation transmissions and consequently result in sparing of the vitality. In grouping, the typical sensor hubs (bunch individuals) rest times are drawn out, while group heads arrange the exercises of its part hubs, again coming about into vitality sparing [3-5]. This movement planning is executed generally through TDMA.

Remote Sensor Networks will be systems that comprise of minor detecting gadgets (down to the span of a grain), called sensor hubs. These sensor hubs are utilized to screen parameters like the temperature of a specific and afterward, transmits these detected data to another sensor hub or some other gadget in the system. Subsequently, to transmit these detected data, the hub requires some measure of vitality. This vitality is provided to the hubs by methods for batteries which perhaps replaceable or non-replaceable and again these batteries may likewise be batterypowered, contingent upon sun-oriented power for reviving. In the majority of the applications, it is beyond the realm of imagination to expect to supplant these batteries, and henceforth these hubs must expend as little power as conceivable amid transmission of information. Thus, the lifetime of the hubs will be expanded which thus will likewise build the lifetime of the system as the lifetime of a system exclusively relies upon the lifetime of the hubs present in the system.

For decrease of vitality utilization in sensor hubs, additionally, grouping encourages information conglomeration at bunch head by diminishing the number of transmitted information bundles. The correspondence in grouping conventions is executed in two stages, the first is intra-bunch, i.e. inside bunches, and the second is between-group, i.e. between the groups and the Base Station. Moreover, the correspondence in a remote sensor system grouping convention can be taken up either by utilizing single bounce transmission or multi-jump steering. The vast majority of the bunching conventions utilize single bounce correspondence for conveying inside the 
group, as the separation between sensors inside the group is generally short, e.g.HEED and LEACH [6-8]. Looks into proposed in writing report that multi-bounce correspondence between the sensor hubs and the group head is more vitality productive than single when the spread misfortune type is high. This is when sensor hubs are sent in thick vegetation areas, or structures, or industrial facilities. In such cases, multi-bounce correspondence is effective in conquering signal engendering troubles. In any case, because the radio disperses vitality in transmission as well as in gathering, coordinate transmission is likewise valuable. Be that as it may, there impedes if there should be an occurrence of direct transmission moreover. It is great to utilize it up to a specific limit remove it as it were. This is because if there should arise an occurrence of transmission separate past edge remove, the vitality cost builds as indicated by the fourth intensity of the separation. As the sensor hubs are vitality obliged, they generally have a restricted transmission go. Along these lines, to expand the system versatility likewise, multibounce correspondence is ideal. If there should be an occurrence of correspondence from the group make a beeline for the BS, on the off chance that BS being far from the sensor field, it is smarter to utilize multi-jump correspondence. There areseveral grouping conventions built up that utilize multi-jump correspondence for accomplishing more vitality productive between bunch correspondences Multi-bounce LEACH, EADC, EDUC, and so on are some such conventions [9].

One of the essential worries in remote sensor systems is a boost of system lifetime because after the system ends up broken, a huge measure of vitality ought not to stay in the hubs, else it is wastage. Many research works have characterized the system lifetime to be the point at which the main hub is dead (FND). The thought behind this suspicion is that every one of the hubs of the system must vanish roughly in the meantime to keep away from the early loss of detecting inclusion, and likely parceling of the system. In any case, as the lifetime necessity is application explicit, considering the primary hub dead as the lifetime definition is not a nonexclusive one. There are distinctive kinds of sensor system applications and in this manner, to take into account diverse application necessities, the lifetime of the system has additionally been assessed at various stages, i.e., when the first hub bites the dust, or a certain level of hubs fizzle. Regardless, it is more critical than organize capacities self-ruling and ensures its activity until its lifetime. In a grouping convention, a $\mathrm{CH}$ is intensely loaded as it needs to perform different assignments, for example, bunch development, information conglomeration, information transmission, and handing-off. Bunch heads in this way devour more vitality when contrasted with non- $\mathrm{CH}$ hubs $[10,11]$. In between group transmission for both the methods of correspondence, single bounce and multihop, there is an inescapable issue of vitality unevenness among sensor hubs. For single bounce correspondence, bunch heads that are far from BS deplete out their vitality principally as a result of the long separation transmission. Be that as it may, when utilizing multi-bounce correspondence in grouping conventions, at that point, the bunch heads close to the base station exhaust their vitality rapidly in light of the additional weight of movement handing-off. This lopsided correspondence stack results in a vitality gap or problem area zone. Because of this, loss 
of detecting inclusion and dividing of the system happen and eventually influence the system execution [12].

In this paper, an endeavor has been made to enhance arrange life expectancy of an EADUC convention utilized in ceaseless observing applications. The EADUC utilizes non-uniform bunching calculation to alleviate the vitality gap issue. The center thought in our proposed plan is that amid the group head choice sub-stage, hubs rivalry sweep task would be founded on not just the separation factor and hub's leftover vitality as is utilized in EADUC, yet additionally a tertiary factor, the number of neighbor hubs. This area data is considered as the bunching parameter to expand arrange life expectancy. Another key thought utilized in our enhanced EADUC convention is amid the determination strategy of activity transferring. The cost engaged with handing-off, regarding vitality, is joined as the measurements for choosing one of the doable hubs as a transfer hub rather than just the separation data utilized in EADUC. The proposed methodology lessens the bunching overhead and in this manner draws out the system lifetime. The execution of our proposed convention is contrasted and the current conventions utilizing system lifetime as the execution metric. In this manner, while structuring a system it is basic to fulfill two imperative criteria, for example, prolongation of system lifetime and vitality proficient information transmission. Consequently, the principle point of this undertaking is to lessen the vitality devoured by the system amid the transmission of information and in the meantime give a vitality productive and dependable information transmission.

\section{Related work}

A prior research work taken up in the field of bunching calculation has been founded on group heads revolution in each cycle and choosing group heads $(\mathrm{CH})$ in light of hubs that have more remaining vitality. The main convention accessible in this class is the low-vitality versatile grouping progressive system (LEACH) convention [13]. It chips away at the guideline of singlebounce correspondence between the base station and the hubs. This makes it temperamental for extensive scale systems. There are different other changed LEACH conventions which are enhancements over the LEACH convention. A portion of the adjusted conventions is a multibounce variation (M-LEACH), LEACH-DT, C-LEACH, and MOD-LEACH. Over the most recent couple of years, scientists have investigated the techniques to palliate the vitality opening issue in various leveled (bunch-based) WSNs. Numerous vitality effective calculations have been produced utilizing equivalent and unequal size bunching systems. Here we consider unequal size grouping just to counter the issue of uneven vitality utilization among sensor hubs of the system. The principal calculation proposed in the class of using unequal group estimate is an unequal bunch measure (UCS) demonstrate, which shapes unequal bunches for facilitating the overburdened bunch heads and guaranteeing better adjusting of vitality dispersal among hubs [14]. The work considered a heterogeneous system and the deterministic organization of group heads at pre-figured areas for controlling the bunch estimate. It accomplished an enhancement of 
$10-30 \%$ over the equivalent bunching size procedure, contingent upon the total effectiveness of $\mathrm{CH}$ hubs. In EEUC, a grouping and circulated calculation is intended for information gathering applications, which proficiently sorts out the system hubs utilizing unequal bunching and multijump directing.

Be that as it may, in this strategy the group head determination is probabilistic and accordingly singular hubs can be delivered Hybrid vitality proficient dispersed (HEED) bunching calculation, where the $\mathrm{CHs}$ are chosen relying on the lingering energies of the hubs and the expense of correspondence for intra-groups. For correspondence in between bunch, utilization of multibounce correspondence comes viable. It is viable in drawing out the lifetime of the hub. It needs in adjusting the heap as hubs near BS bite the dust rapidly. Grouping is one of the answers for better vitality preservation during the time spent correspondence in WSN [15]. Another grouping calculation named Unequal Clustering Algorithm (UCA) is actualized to annihilate the overheads of the bunch head which is arranged close to the sink as a result of the movement from alternate CHs. Improved Unequal Clustering Algorithm (EUCA), endeavors to decrease the movement between bunching by killing the opposition between the hubs contending to be the Cluster head $(\mathrm{CH})$.

Recreation thinks about unmistakably demonstrate the proficiency of EUCA as far as vitality utilization. Bringing down the overheads in gathering the information is utilized to diminish the vitality gap issue. Unequal measured groups are utilized for making the bunches in this convention. Sink Mobility-based Energy Balancing Unequal Clustering (SMEBUC) is a convention with a circulation of hubs, which chooses its Cluster Head which has the most extreme vitality among every one of the hubs. The hubs are dispersed into bunches of different sizes with an enhanced calculation called the Shuffled Frog Leaping Algorithm (SFLA) [16-18]. The covetous calculation is utilized to choose the hand-off hub which is ideal for the transmission of information between the Cluster Head and Sink hub. Another technique, a vitality-driven unequal bunching convention (EDUC), thinks about a dispersed unequal grouping calculation and a vitality-driven versatile bunch head turn strategy. In this the vitality devoured in group head pivot is limited by enabling a hub to be bunch head just once amid the system lifetime. Consequently, by decreasing the vitality cost utilized in bunch head turn, it accomplishes vitality proficiency. In any case, it has the restriction that it is helpful for singlebounce arranges as it were $[19,20]$.

In the proposed work, we are endeavoring to enhance the current convention in particular Energy Distributed Unequal Clustering (EDUC) convention. We are utilizing a multi-bounce way to deal with taking care of the vitality gap issue. The vitality gap issue is where the bunches close to the Base station (BS) lose more vitality than the groups which are sitting far from the Base station (BS). Another methodology is executed that will make the system more productive by choosing an exchange group head from the rest of the hubs after every transmission. 


\section{Methodology}

Various other mechanisms used for minimizing the energy consumed in WSN are described here.

\section{Energy Model}

It is one of the required things which is utilized to calculate the sensing network performance. A detailed description of the various mechanisms is described below.

\section{Co-operative model}

The energy consumed for cooperative communication is essentially a result of information transmission. As referenced before the aggregate vitality required for the transmission of information relies upon two primary parameters [21]. The total energy for cooperative communication relies upon the accompanying variables for effective transmission,

No. of nodes

$>$ Probability in broadcasting

$>$ Probabilityco-operative transmission

$>$ Distance in broadcasting

For CC transmission the total energy is comprised of two types namely,

$>$ Local transmission - source node to cooperative nodes.

$>$ Long haul transmission - from c-nodes to the sink.

The equation for $\mathrm{CC}$, local transmission $\left(e_{b}\right)$, haul transmission $\left(e_{c t}\right)$ and data transmission $\left(e_{t}\right)$ is given below,

$$
\begin{array}{ll}
e_{c c}=e_{b}\left(m_{c n}, q_{c}\right)+e_{c n}\left(m_{c n}, q_{d}\right) & \text { Equation 1 } \\
e_{b}\left(s_{c}^{l}, q_{c} m_{c n}\right)=e_{t}\left(s_{c}^{l}, q_{c}, 1\right)+\left(m_{c n}-1\right) e_{s} & \text { Equation 2 } \\
e_{c t}\left(d^{k}, q_{c}, m_{c n}\right)=e_{t}\left(d^{k}, q_{c}, m_{c n}\right)+e_{s} & \text { Equation 3 } \\
e_{t}\left(d^{k}, q_{e}, m_{c n}\right)=\left(m_{o} \times \frac{d^{k}}{\frac{1}{m_{c}}}\right)+\left(\frac{q_{c t}}{s_{c}}\right) & \text { Equation 4 }
\end{array}
$$


Where $k$ denotes the path-loss exponent whose value depends on the environment through which the data is transmitted.

\section{Coalition and cluster-based Data Transmission model}

Another sort of cooperative communication is the Coalition and cluster-based information transmission instrument. The system comprises $\mathrm{n}$ number of sensor hubs and one sink, (for example, a base station) [22]. The system comprises of $\mathrm{M}$ number of alliances and $n i$ is the quantity of $\mathrm{SN}$ in the ith alliance, with the end goal that the comparing condition is given as,

$$
\begin{array}{ll}
\sum_{i=1}^{n} m_{i}=m & \text { Equation } 5
\end{array}
$$

The energy consumption in cluster-based is divided into two parts namely Intra and inter-cluster data transmission. The energy consumed by this is given below,

$$
\begin{array}{ll}
e_{g}^{\prime}=\frac{h_{o}}{s}\left(m_{i}-1\right)\left[\frac{q}{\gamma_{c}^{i^{2}}}+q_{s}\right] & \text { Equation } 6 \\
e_{t}^{\prime}=\frac{h_{i}}{s}\left[\frac{q}{\gamma_{i k}^{2}}+q_{s}\right] & \text { Equation } 7
\end{array}
$$

The total energy consumed by the cluster-based protocol is given below,

$$
\begin{array}{ll}
e_{\text {total for } \text { cluter }}=e_{g}^{\prime}+e_{t}^{\prime} & \text { Equation } 8
\end{array}
$$

An essential issue in remote sensor systems is to augment the system lifetime under given vitality requirements. To accomplish the objective, vitality utilization must be very much adjusted among hubs. Hence, the essential goal of this paper is attempting to astutely plan the bunching and multihop steering plan to broaden the system lifetime. We receive both the revolution of group heads and picking bunch heads with more lingering vitality. Moreover, we present a novel unequal grouping component which is a compelling technique to manage the problem areas issue. It can keep the untimely making of vitality gaps in remote sensor systems [23].

\subsection{Proposed method}




\section{Unequal clustering algorithm and inter-cluster multi-hop routing}

In this system, the base station communicates a "welcome" message to all hubs at a specific power level. By thusly every hub can figure the surmised separation to the base station dependent on the got flag quality. It does not just encourage hubs to choose the correct power level to speak with the base station, yet additionally causes us to deliver bunches of unequal size. Point-by-point depictions of the unequal grouping calculation is introduced in the accompanying segment.

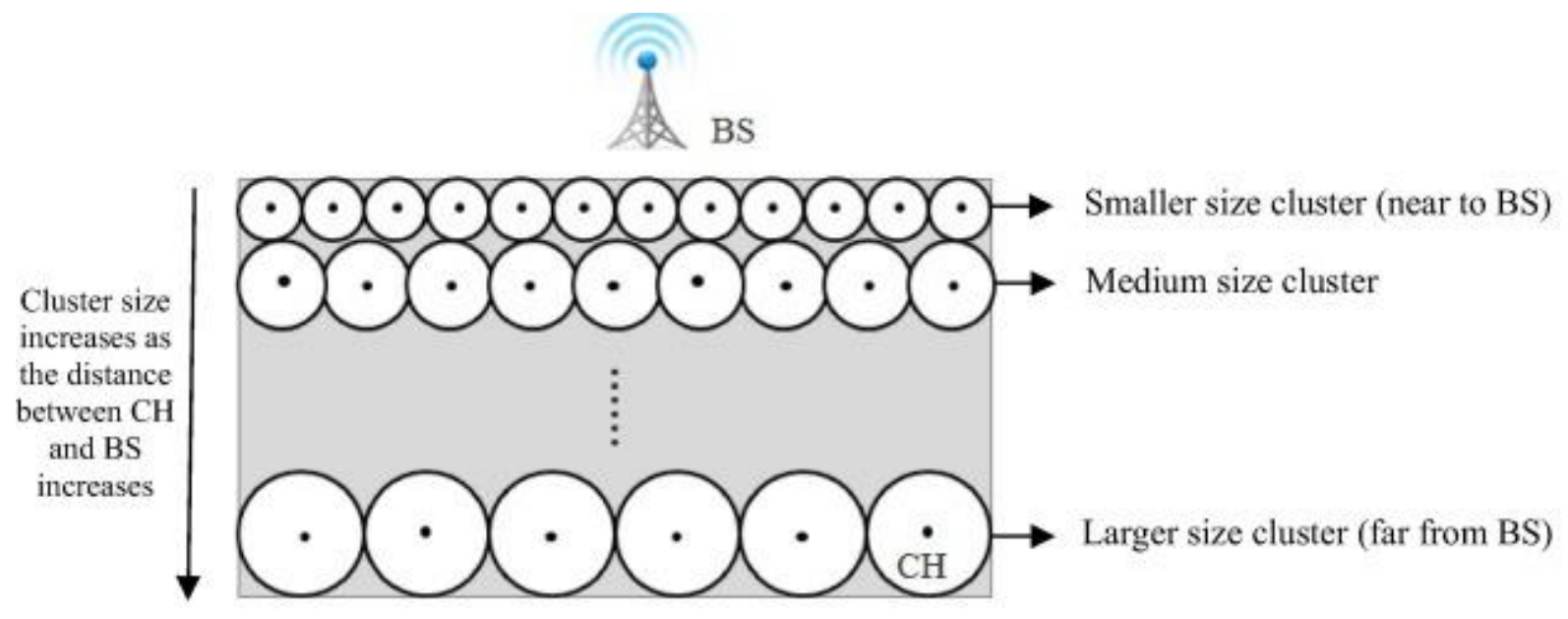

Figure 1 Basic architecture of unequal clustering

Bunching a remote sensor arrange implies apportioning its hubs into groups, everyone with a group head and some conventional hubs as its individuals. The undertaking of being a group head is pivoted among sensors in every datum assembling round to disperse the vitality utilization over the system. EEUC is an appropriated bunch heads-focused calculation, where group head choice is principally founded on the lingering vitality of every hub. The pseudo-code for a discretionary hub is given underneath. 


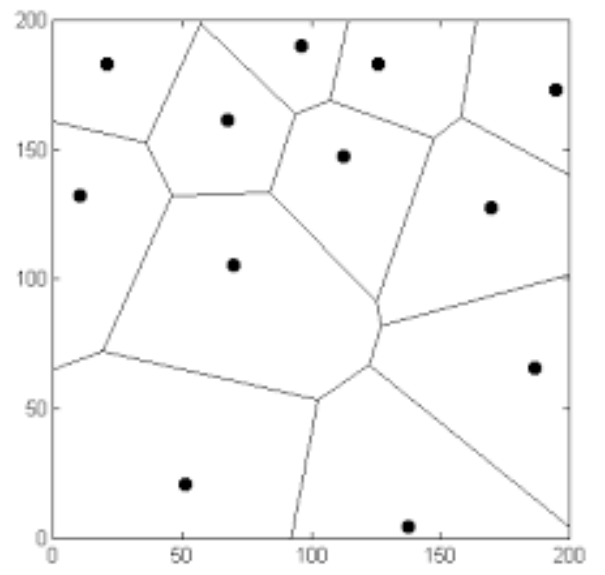

Figure 2 Clusters formed as a cell around heads

\section{Algorithm for Cluster head Selection}

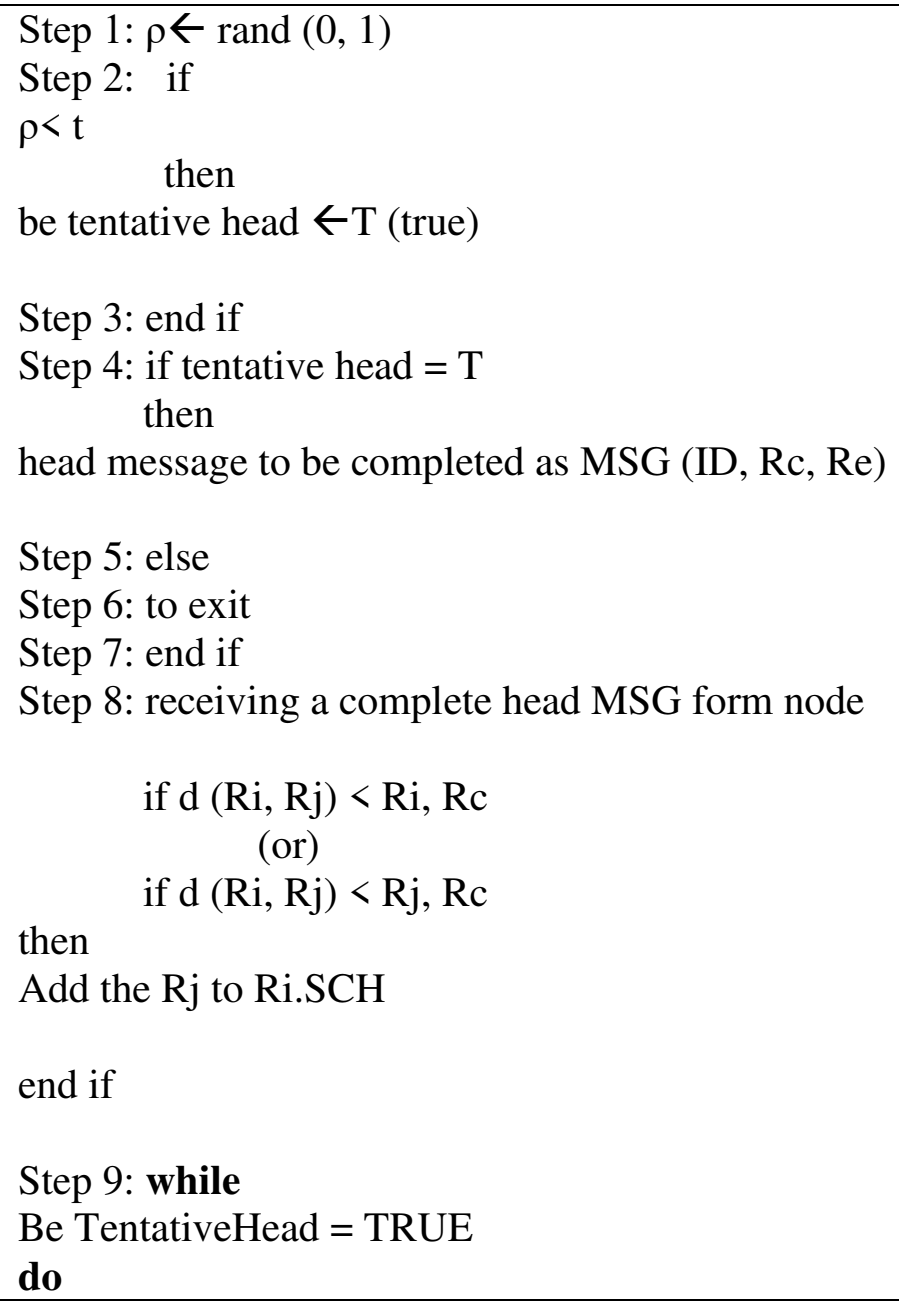


Step 10: if Ri. Se> Rj.Se, then

Finally Head MSG and then exit

end if

on receiving the final Hmsg from node $\mathrm{Rj}$

Step 11: if $\mathrm{Rj} € \mathrm{Ri}$.SCH, then quit election msg and then exit end if

on receiving $\mathrm{Q}$ msg from node $\mathrm{Rj}$

Step 12: if $\mathrm{Rj} € \mathrm{Ri}$.SCH, then

Remove Rj from Ri.SCH

end if

end

while

\section{Inter-cluster Multihop Routing}

At the point when group heads convey their information to the base station, each bunch headfirst totals the information from its group individuals, and afterward sends the parcel to the base station using multi-hop correspondence. In some proposed calculations like PEGASIS, transfer hubs can total the approaching bundles from different groups together with their very own parcels [24-26]. This supposition is eccentric because the level of detected information connection between's various bunches is similarly low. In this paper, hand-off hubs don't total the approaching parcels. The steering issue here varies significantly from that of conventional specially appointed remote systems due to the many-to-one movement design. Then again, neither inquiry-driven nor occasion-driven directing conventions for remote sensor systems can be connected to the group heads overlay. Hence, we plan an upgraded multi-bounce steering convention for the between bunch correspondence.

\subsubsection{Improved protocol mechanism}

The bunching technique utilized is comparable in activity to typical EDUC convention. The convention works in rounds. After hubs are sent, every hub initially figures its separation from a base station. For this, BS communicates a flag, which is heard by all hubs. Based on the got flag quality, every hub approximates its separation to BS. Each round contains a bunch set-up stage and relentless state stage in which information transmission happens. The set-up stage is further sub-isolated into three sub-periods of terms T1, T2 and T3 individually. The primary sub-stage is 
the neighbor hub data gathering. Toward the start of the data accumulation sub-stage, every hub communicates a Node_Msg, which contains its lingering vitality alongside its id. Every one of the hubs, which are in its radio range, gets the Node_Msg from every one of its neighbors.

The average residual energyof the cluster for each according to the below equation,

$$
e_{\text {average value }}=\frac{\sum_{i=1}^{n} R_{i}, e_{S}}{m_{c}} \text { Equation } 9
$$

The upgraded EAUC conspire depends on the EADUC convention; in any case, as opposed to the EAUC, it utilizes an alternate rivalry range rule for delivering unequal groups. In the first EAUC convention, in the articulation for rivalry span, just the separation between the hubs and the BS, and the remaining vitality of the hubs is considered. To represent the cost engaged with accumulation, the proposed plan additionally thinks about the number of neighbors, notwithstanding the over two elements, while choosing the opposition radii. The opposition sweep for the proposed plan is a component of separation to the BS, the leftover vitality of $\mathrm{CH}$, and the number of neighbor hubs. Hubs with moderately higher leftover vitality, more prominent separation from the BS, and a lower number of neighbor hubs ought to have a bigger rivalry range.

For achieving it, we used the formula given below,

$$
R_{C}=\left[1-\gamma\left(\frac{D_{\max }-D\left(R_{i}, B S\right)}{D_{\max }-D_{\min }}\right)-\beta\left(1-\frac{e_{r}}{e_{\max }}\right)+\alpha\left(1-\frac{R_{i}(m c)}{m c_{\max }}\right)\right] \times R_{\max } \quad \text { Equation } 10
$$

The possibility of incorporation of neighborhood data for group head choice alongside accessible vitality and separation to the sink is utilized in some current conventions, viz. an unequally grouped multi-bounce directing convention (UCMR), and crossbreed unequal bunching with layering convention (HUCL). Nonetheless, in the UCMR and HUCL conventions, the quantity of neighbors isn't considered while registering the opposition radii. The computation of rivalry radii in these papers utilizes the separation parameter just as is utilized in a vitality productive unequal bunching system (EEUC) or an unequal group-based steering convention (UCR).

The group heads transmit the information parcel to the BS either specifically, or through transferring. On the off chance that the separation from a particular bunch make a beeline for BS is more prominent than limit remove (dist_th), between-group correspondences are completed; generally, coordinate transmission is executed. For between bunch correspondence, the determination of group head as next jump hub (transfer hub) is then drawn. In the first EAUC 
convention, hand-off hub determination is done as one of the neighbor hubs from the competitor sending set by a parameter Erelay.

This Erelay parameter is computed as given below,

$$
e_{\text {realy }}=D^{2}\left(R_{j}, R_{i}\right)+D^{2}\left(R_{j}, B S\right)
$$

In the proposed plan, for between-group correspondence processes, each bunch headfirst communicates a message involving its hub id, leftover vitality, tally containing the number of group individuals, and separation to the base station. To additionally enhance the execution, the group head turn isn't done in each round. Rather once the bunch set up is acquired, it is held for a couple of rounds.

In one round of convention tasks, the information transmission stage runs several times. For this, the unfaltering state includes various real spaces and for each significant opening further contains several smaller than usual openings. In every less space, the entire procedure of information transmission elimination is conveyed. Amid the last smaller than normal space, the part hubs send their remaining vitality alongside the information. After one noteworthy opening is finished, the group head turns inside the bunch limit is completed. The old group head gets supplanted by another bunch head in a similar group contingent upon the leftover vitality of the hubs and the separation from the present bunch head. The part hub having higher outstanding vitality and least separation from it is picked as the new bunch head. The old bunch heads hands over the individual's rundown to the new group head. At the point when the new group head determination and handover happens, another significant opening starts. After the fruition of the number of real spaces, another round of convention runs involving the setup stage and consistent state stage.

\section{Protocol and performance analysis}

This segment displays the examination of the unequal bunching calculation. As per the Algorithm, the bunch head determination process is message-driven. To choose whether it will be a group head or a conventional hub in the Algorithm, each speculative hub Rj sits tight for the choice of every hub $x$ in its $\mathrm{SCH}$ with the end goal that $\mathrm{x}: \mathrm{SE}>\mathrm{Ri}: \mathrm{SE}$.

How about we allude to beneath figure to pick up a piece of knowledge into the issue of holding uptime. Assume s1: SE < s2: SE < s3: SE < s4: SE < s5: SE, i.e., they frame a gradual vitality chain. The accompanying occasions will happen in a steady progression. The model demonstrates the holding up time relies upon the longest monotone vitality chain. Be that as it 
may, because the lingering vitality of conditional bunch heads is appropriated haphazardly, the more drawn out a monotone vitality chain is, the littler the likelihood is. In some comparable work, the creator examines a comparable issue and brings up that the holding up time relies upon the vitality topology of the system as opposed to on the number of hubs in the system.

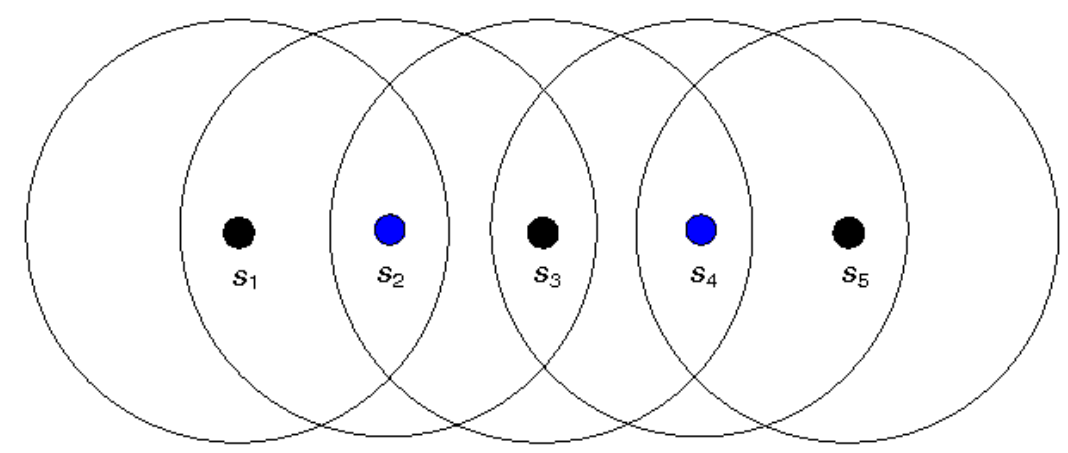

Figure 3 Five nodes - monotonic energy chain

\section{Result and discussion}

In this segment, we assess the execution of the upgraded EEUC system through simulation. First, we think about the group head qualities of the unequal bunching calculation, at that point we explore how improved EEUC balances the vitality utilization of the bunch heads and in this manner delays the system lifetime.

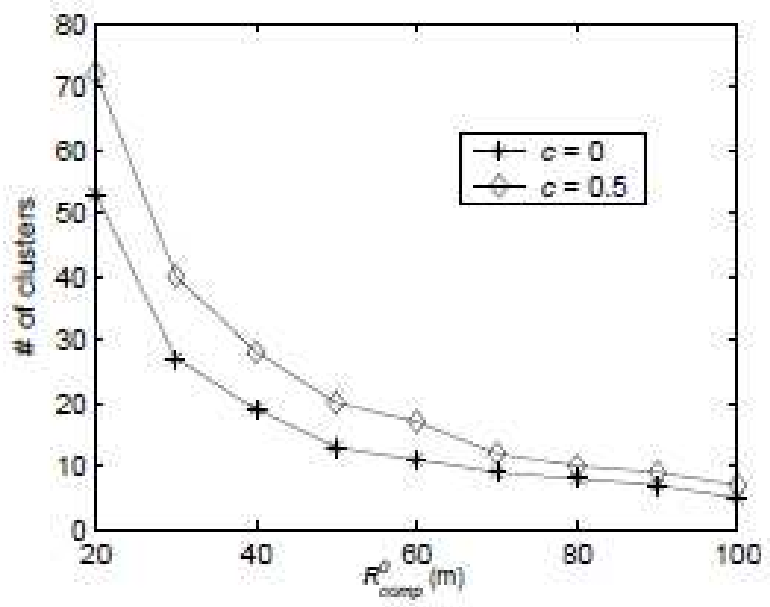

Figure 4 Number of clusters generated

We contrast improved EEUC and LEACH and HEED. In our usage of HEED, multihop steering is utilized amid bunch heads conveying the information to the base station as indicated by a few 
references. We likewise run broad tests to decide the ideal number of bunches to use in LEACH, and the ideal group span to use in HEED.

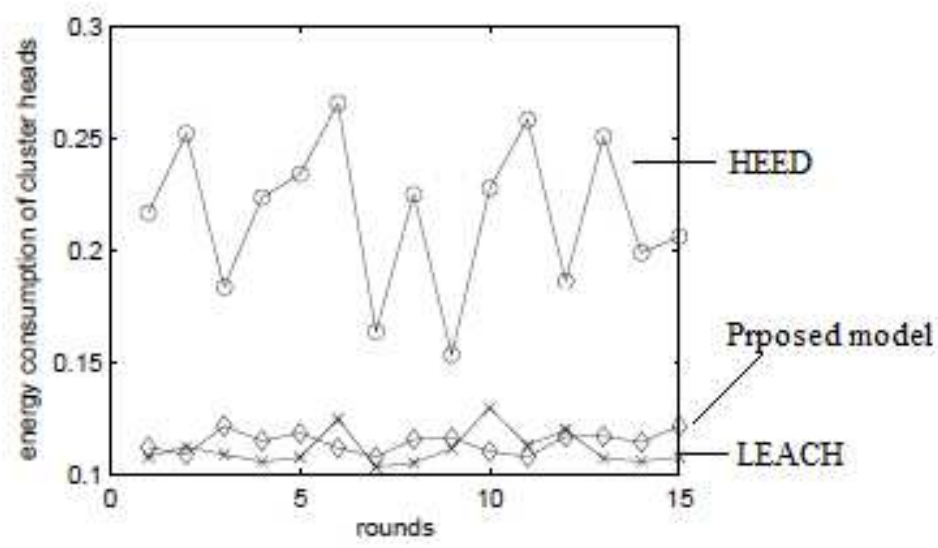

Figure 5 Amount of energy spent by clusters

The fundamental point of this work is to decrease the vitality expended amid the transmission of information from source hub to goal hub. Our work is to break down the execution of the system and the vitality utilization, by fluctuating at least one element. Contingent upon how these elements influence the transmission vitality we discover the ideal incentive for these elements with the end goal that the transmission of information is done in a vitality proficient and in a solid way.

\section{Simulation environment for various scenarios}

For simulation, we chose different three scenarios as follows,

\section{$1^{\text {st }}$ scenario:}

Forover an area of $200 \times 200 \mathrm{~m}^{2} 120$ nodes are uniformly deployedin the below figure 


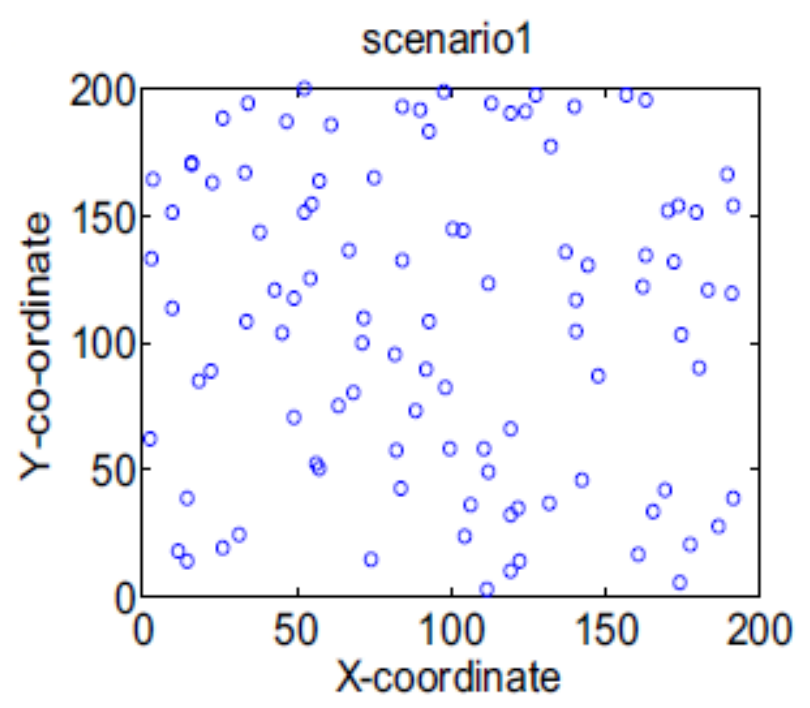

Figure 6 Network topology $-1^{\text {st }}$ scenario

\section{$2^{\text {nd }}$ scenario:}

On the right side of the sensor field, more nodes are grouped along with non-uniformly deployed 120 nodes near the base station over an area of $200 \times 200 \mathrm{~m}^{2}$ as shown below.

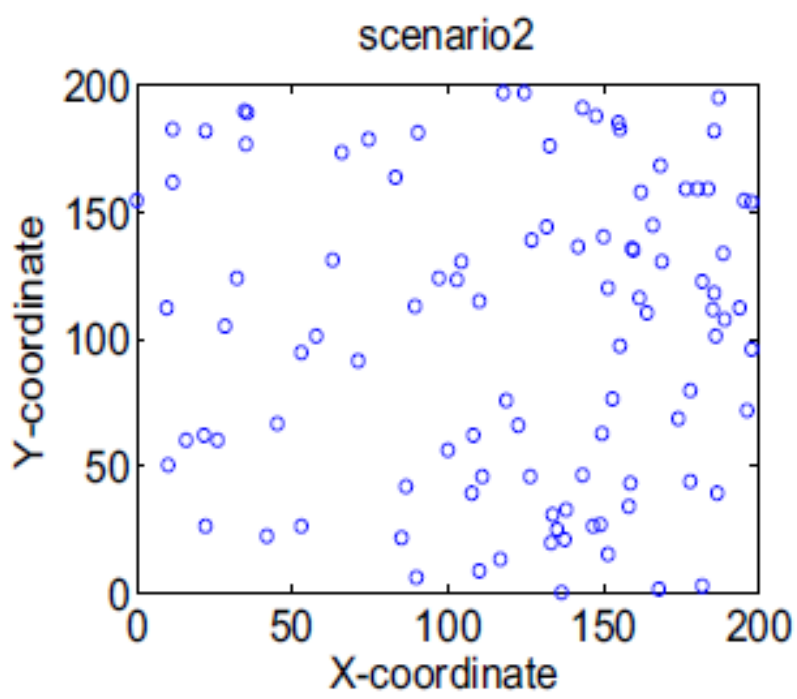

Figure 7 Network topology $-2^{\text {nd }}$ scenario

\section{Scenario 3:}

The various number of sensor nodes are grouped along with non-uniformly deployed 120 nodes in the left region. 


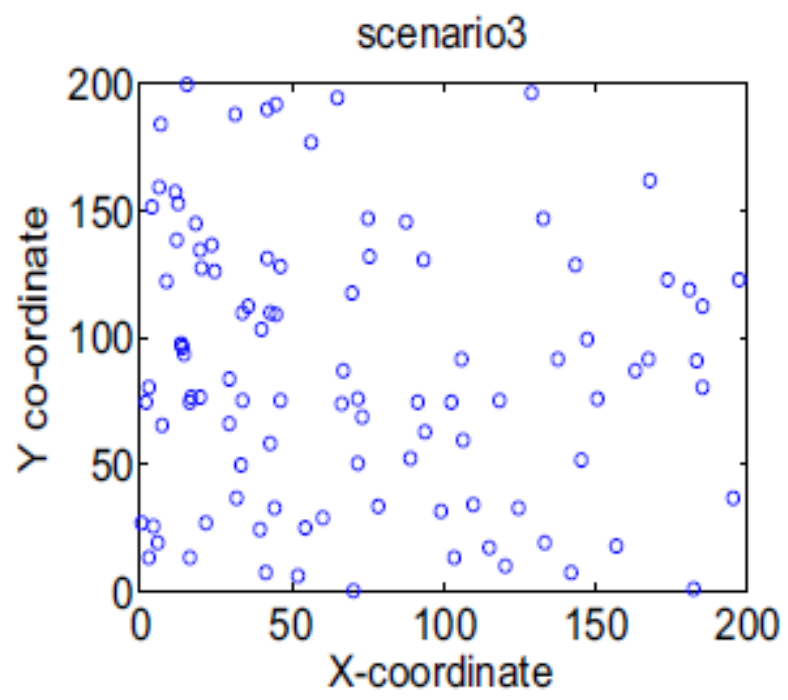

Figure 8 Network topology $-3^{\text {rd }}$ scenario

To watch the impact of the proposed strategy for registering rivalry span and transferring and the method of division of information transmission stage into major and smaller than expected spaces independently, the consequences of the proposed convention, the improved EDUC, are appeared in two stages in the consequent segments. In the primary execution, to be specific enhanced EDUC 1, the proposed convention utilizes the technique for grouping and transferring just without fusing the division of information transmission stage. In the second execution, to be specific enhanced EDUC 2, the strategy for grouping and handing-off alongside the procedure of division of information transmission stage is joined. It is seen that the remaining vitality of hubs in the system diminishes at nearly a similar rate in the event of situations 1 and 2 . In any case, the aggregate outstanding vitality of the system is less if there should be an occurrence of situation 3 when contrasted with situations 1 and 2. This is a result of bigger vitality utilization by hubs of the system in situation 3. Also, this is because the district close to the BS is meager in situation 3 and also the stability of our clustering algorithm shows the distribution of the number of clusters below figure 10 . 


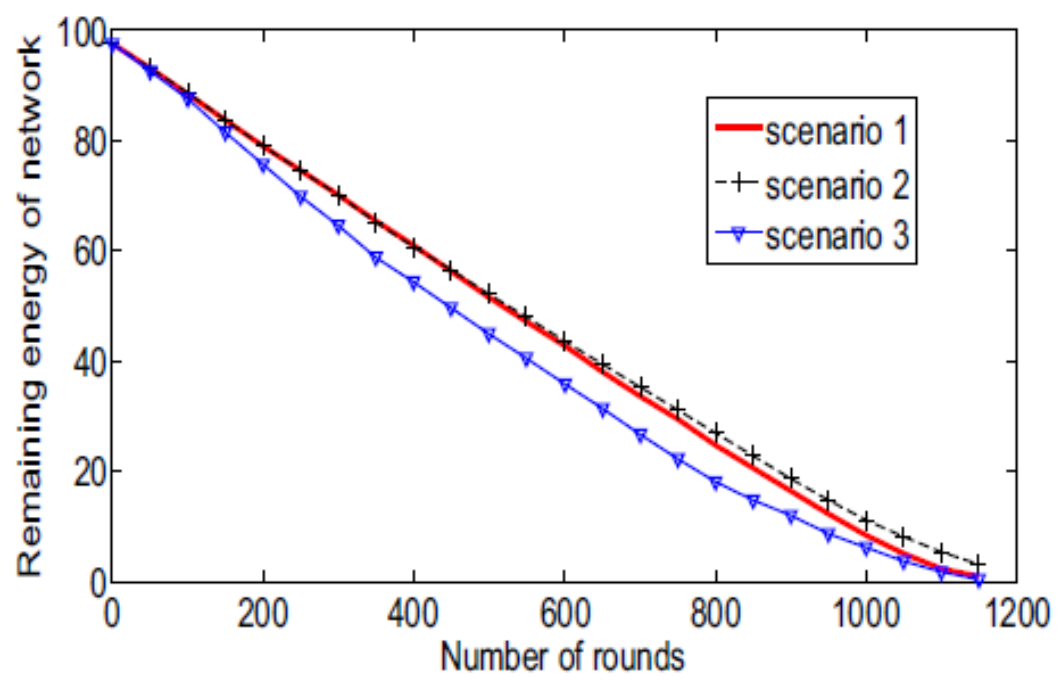

Figure 9 Residual energy average in various rounds

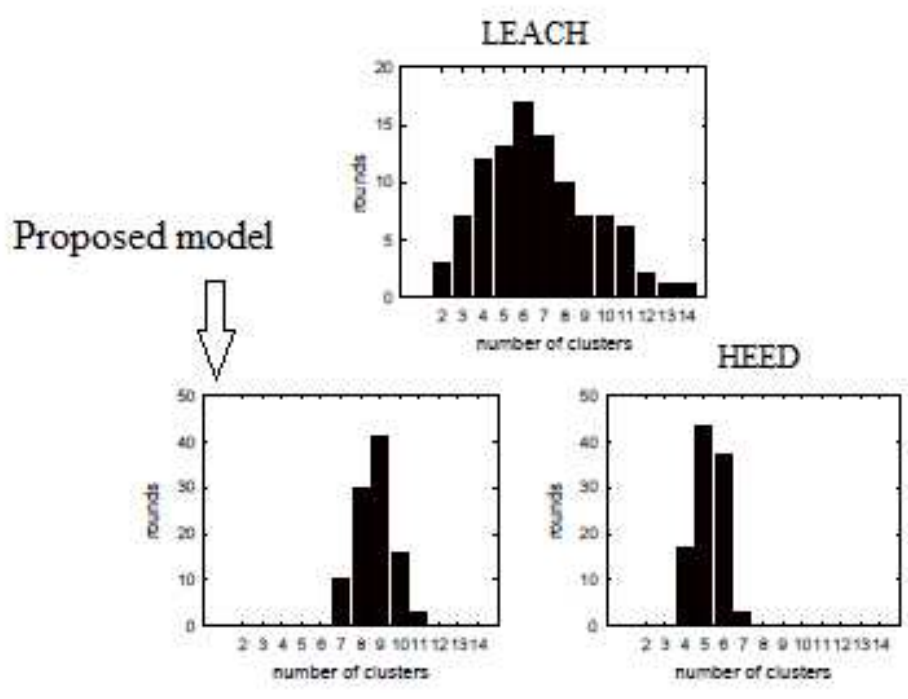

Figure 10 Number of clusters in each rounds

\section{Energy Efficiency}

In this part, we examine the energy effectiveness of upgraded EEUC. In the first place, we think about the measure of vitality spent by group heads in three calculations. 15 rounds of recreations are examined and the measure of aggregate vitality spent by all group heads is appeared in the beneath figure. 


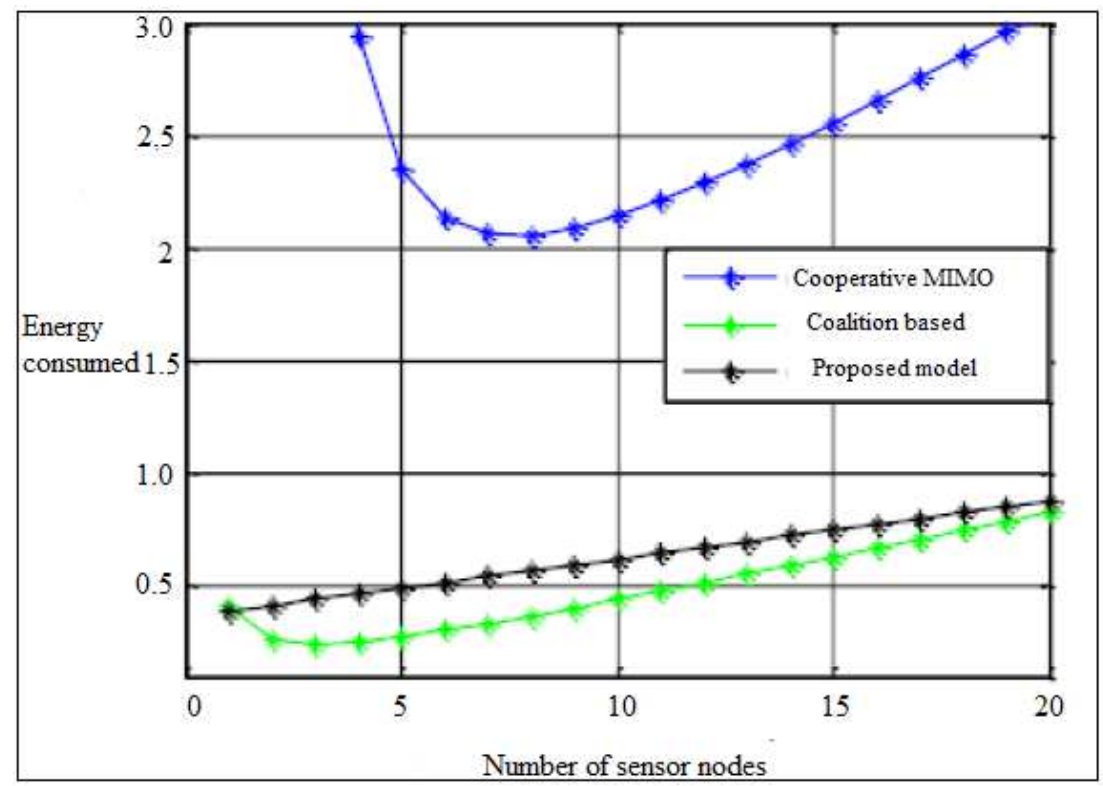

Figure 11 Energy comparison between other algorithms

The vitality devoured by cluster heads per round in overhauled EEUC is much lower than that in $\mathrm{LEACH}$, and is about proportional to that in HEED. Since bundle heads send their packages to the base station by methods for a single bounce in $\mathrm{LEACH}$, the imperativeness use is significantly higher. In addition, because the allocation of picked amass heads is wild in LEACH, there is a fundamental assortment of essentialness usage of the bundle heads. In EEUC and HEED, gather heads transmit their data to the base station by methods for multihop, henceforth a considerable measure of vitality is saved.

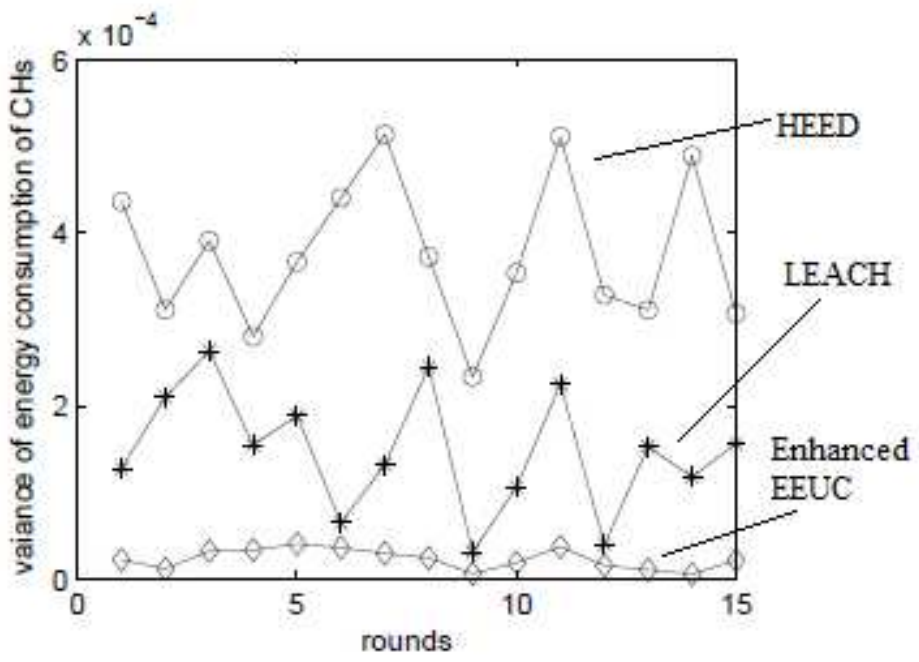

Figure 12cluster heads - in case of energy spent 
Because of the strength of group heads topology in the two techniques, the measure of vitality spent by bunch heads is nearly the equivalent in each round. Third, we check the unequal grouping system, in reality, expand the system time. The outcome appears in the above figure, which legitimizes our unequal bunching component. At last, we look at the vitality effectiveness by contrasting the proposed and other existing calculations by inspecting the system lifetime. The above demonstrates the number of sensor hubs still alive over the recreation time. Upgraded EEUC unmistakably enhances the system lifetime (both the time until the point that the principal hub kicks the bucket and the time until the point that the last hub bites the dust) over LEACH and HEED. In HEED, speculative group heads are haphazardly chosen dependent on their leftover vitality. Subsequently, sensors with low lingering vitality can even now moved toward becoming bunch heads since it utilizes the intra-group correspondence cost to choose the last bunch heads. Consequently, a few hubs bite the dust too before HEED. This is evading in upgraded EEUC because vitality utilization is very much adjusted among hubs. The little interim between the time until the point when the main hub passes on and the time until the point when the last hub kicks the bucket infers that EEUC has effectively tackled the problem areas issue.

\section{Conclusion}

In this paper, we have presented enhanced energy distributed unequal clustering protocol (Enhanced EDUC) to improve the lifespan of WSN. The problem areas issue shows up while utilizing the multihop steering in a bunching approach. We contend that both the revolution of group heads and the measurement of remaining vitality are not adequate to adjust the vitality utilization over the system. To address the issue, we initially acquaint an unequal grouping component with equalization of the vitality utilization among bunch heads. The non-uniform grouping approach has been misused in this work. The clusters shaped are of unequal size by utilizing uneven rivalry range. The clusters nearer to the base station have littler size than groups that are far from the BS. The hubs are allotted uneven rivalry range through the utilization of various components, viz. the separation to BS, the lingering vitality, and the number of neighbors. Subsequently, the vitality utilization among the group head hubs is all the more adequately adjusted. The reproduction results demonstrate that arrange life expectancy is delayed successfully in every situation contrasted with the EEUC and HUCL conventions. The result of this investigation will be valuable for taking care of the vitality gap issue in information gathering systems.

\section{Declarations}

1. Funding Not Applicable

2. Conflicts of interest/Competing interests

There is no conflict of interest from all the authors in the manuscript. 

3. *Availability of data and material
Not Applicable
4. *Code availability (software application or custom code) Not Applicable
5. *Authors' contributions
T Ashok - Overall concepts, literature survey, Working and ideology, Results development R Prabhakaran - Supervising, Proof editing

\section{References}

1. F. Akyildiz, W. Su, Y. Sankarasubramaniam, and E. Cayirci, "A Survey on Sensor Networks", IEEE Communications Magazine, vol. 40, no. 8, pp. 102-114, 2002.

2. B. Krishnamachari, D. Estrin, and S. Wicker, "The Impact of Data Aggregation in Wireless Sensor Networks", in Proceedings of IEEE Int'lConference on Distributed Computing Systems Workshops (ICDCSW), 2002, pp. 575-578.

3. V. Mhatre and C. Rosenberg, "Design Guidelines for Wireless Sensor Networks: Communication, Clustering and Aggregation", Ad Hoc Networks, vol. 2, no. 1, pp. 45-63, 2004.

4. W. Heinzelman, A. Chandrakasan, and H. Balakrishnan, "An Application-Specific Protocol Architecture for Wireless Microsensor Networks", IEEE Transactions on Wireless Communications, vol. 1, no. 4, pp. 660-670, 2002.

5. O. Younis and S. Fahmy, "HEED: A Hybrid, Energy-Efficient, Distributed Clustering Approach for Ad Hoc Sensor Networks", IEEETransactions on Mobile Computing, vol. 3, no. 4, pp. 660-669, 2004.

6. S. Lindsey, C. Raghavendra, K. M. Sivalingam, "Data Gathering Algorithms in Sensor Networks Using Energy Metrics", IEEE Transactionson Parallel and Distributed Systems, vol. 13, no. 9, pp. 924-935, 2002.

7. W. Choi, P. Shah, and S. K. Das, "A Framework for Energy-Saving Data Gathering Using Two-Phase Clustering in Wireless Sensor Networks", in Proceedings of Int'l Conference on Mobile and Ubiquitous Systems: Networking and Services (MOBIQUITOUS), 2004, pp. 203-212.

8. S. SoroandW. Heinzelman, "Prolonging the Lifetime ofWireless Sensor Networks via Unequal Clustering", in Proceedings of the 19th IEEEInternational Parallel and Distributed Processing Symposium (IPDPS), 2005.

9. M. Ye, C. F. Li, G. H. Chen, and J. Wu, "EECS: An Energy Efficient Clustering Scheme in Wireless Sensor Networks", in Proceedings ofIEEE Int'l Performance Computing and Communications Conference (IPCCC), 2005, pp. 535-540.

10. C. Intanagonwiwat, R. Govindan, and D. Estrin, "Directed Diffusion: a Scalable and Robust Communication Paradigm for Sensor Networks", in Proceedings of ACM Mobile Computing and Networking (MOBICOM), 2000, pp. 56-67.

11. C. Schurgers and M. B. Srivastava, "Energy Efficient Routing in Wireless Sensor Networks", in Proceedings of IEEE Military CommunicationsConference (MILCOM), 2001, vol. 1, pp. 357-361

12. V. Mhatre, C. Rosenberg, Homogeneous vs. heterogeneous clustered networks: a comparative study, Proc. IEEE, ICC 6 (2004) 3646-3651. 
13. C.Y. Chang, H.R. Chang, Energy aware node placement, topology control and MAC scheduling for wireless sensor networks, Comp. Netw. 52 (2008) 2189- 2204.

14. X. Gu, J. Yu, D. Yu, G. Wang, Y. Lv, ECDC: an energy and coverage-aware distributed clustering protocol for wireless sensor networks, Comp. Electr. Eng. 40 (2014) 384-398.

15. J. Mao, Z. Wu, X. Wu, A TDMA scheduling scheme for many-to-one communications in wireless sensor networks, Comp. Commun. 30 (2007) 863-872.

16. P. Ayona, A. Rajesh, Investigation of energy efficient sensor node placement in railway systems, Eng. Sci. Technol. Int. J. (2015), doi: 10.1016/j.jestch.2015.10 009.

17. V. Kaundal, A.K. Mondal, P. Sharma, K. Bansal, Tracing of shading effect on underachieving SPV cell of an SPV grid using wireless sensor network, Eng. Sci. Technol. Int. J. 18 (2015) 475-484.

18. S. Bandyopadhyay, E.J. Coyle, An energy efficient hierarchical clustering algorithm for wireless sensor networks, IEEE INFOCOM, 2003, pp. 1713- 1723.

19. H.M. Ammari, S.K. Das, Promoting heterogeneity, mobility, and energy-aware Voronoi diagram in wireless sensor networks, IEEE Trans. Parall. Distr. Syst. 19 (7) (2008) 9951008.

20. D. Vass, Z. Vincze, R. Vida, A. Vidacs, Energy efficiency in wireless sensor networks using mobile base station, EUNICE 2005: Networks and Applications towards a Ubiquitously ConnectedWorld, 2005, pp. 173-186.

21. J. Li, P. Mohapatra, An analytical model on the energy hole problem in manyto- one sensor networks. Proc. of IEEE Vehicular Technology Conf., Fall 2005, pp. 2721-2725.

22. J. Wu, Y. Qi, G. Wang, Q. Guo, X. Gu, An energy aware distributed unequal clustering protocol for wireless sensor networks, Int. J. Distrib. Sens. Netw. (2011) doi:10.1155/2011/202145.

23. J. Li, P. Mohapatra, Analytical modeling and mitigation techniques for the energy hole problem in sensor networks, Pervasive Mob. Comput. 3 (2007) 233-254.

24. J. Jia, X. Wu, J. Chen, X. Wang, Exploiting sensor redistribution for eliminating the energy hole problem in mobile sensor networks, Eurasip J. Wirel. Commun. Netw. 1 (2012) 1-11.

25. L. Malathi, R.K. Gnanamurthy, K. Chandrasekaran, Energy efficient data collection through hybrid unequal clustering for wireless sensor networks, Comp. Electr. Eng. (2015) 1-13.

26. L. Qing, Q. Zhu, M. Wang, Design of a distributed energy-efficient clustering algorithm for heterogeneous wireless sensor networks, Comp. Commun. 29 (2006) 2230-2237. 